\title{
An Experimental-Numerical Combined Method to Determine the True Constitutive Relation of Tensile Specimens after Necking
}

\author{
You-de Wang, Shan-hua Xu, Song-bo Ren, and Hao Wang \\ School of Civil Engineering, Xi'an University of Architecture and Technology, Xi'an 710055, China \\ Correspondence should be addressed to You-de Wang; yord.w@sohu.com
}

Received 23 December 2015; Revised 1 April 2016; Accepted 19 April 2016

Academic Editor: Steve Bull

Copyright (C) 2016 You-de Wang et al. This is an open access article distributed under the Creative Commons Attribution License, which permits unrestricted use, distribution, and reproduction in any medium, provided the original work is properly cited.

\begin{abstract}
To obtain the material true constitutive relation of tensile specimens after necking, we proposed an experimental-numerical combined method (ENM) based on the simple tension test results and finite element analysis (FEA). An iterative scheme was used to minimize the errors between the simulated and experimental load-displacement curves by modifying the imported stressstrain data step by step, and the true stress was determined when the error was less than a given infinitesimal value. In addition, we developed a special program to implement this algorithm automatically and save operating time. As a verification, the true stress-strain curves obtained by the traditional analytical method (TAM) and ENM were compared and employed to analyze the large deformation behavior of both cylindrical and rectangular specimens. The results showed that ENM was applicable for both specimens and could achieve an adequate description of the mechanical response of the materials after necking formation more effectively.
\end{abstract}

\section{Introduction}

The simple uniaxial tension test with employing an extensometer is the most common and fundamental way to evaluate the constitutive relation of metals and extract other mechanical properties such as the elastic modulus, yield strength, tensile strength, and elongation [1]. However, it is well-known that the true constitutive relation during the nonuniform deformation process up to fracture, which is essential in large deformation analysis and fracture failure prediction [2-5], cannot be directly achieved from the test load-displacement $(F-\Delta L)$ curve. Most materials exhibit the same deformation sequences: normally uniform elongation followed by diffuse necking and then localized necking in the form of crossed intense-shear bands (for rectangular specimens with low cross section aspect ratio and strong plastic hardening ability), damage nucleation, and finally fracture [6]. These sequences of deformation are illustrated in Figure 1(a). At small strains, that is, elastic or early plastic regime, the deformation may be assumed, without much loss of accuracy, to deform homogeneously along its length and throughout the whole cross section. Consequently, it allows for a convenient way to measure the extension of a certain gauge length with a so-called extensometer and calculate the value of stress and strain, which can be expressed as the ratio of total force to cross section area and the ratio of displacement to gauge length, respectively. However, the problems will arise when the strain reaches a critical value and the state of specimen goes into another regime, that is, diffuse necking. Structural plastic instability originated from nonuniformities makes it impossible to balance the external loads by a uniform uniaxial stress, so that a multiaxial stress state (see Figure 1(d)) occurs at the narrowest cross section within the necking region [7], which will also limit the accuracy and reliability of experimental measured stress data. In addition, the highly concentrated strain field within necking region (see Figure 1(b)) and the elastic unloading in regions outside the necking (see Figure 1(c)) $[3,8]$ render the conventional extensometry techniques virtually useless for the axial strain measurement inside the neck, because this method measures only the average strain over the gauge length. Another issue that should be mentioned is the 


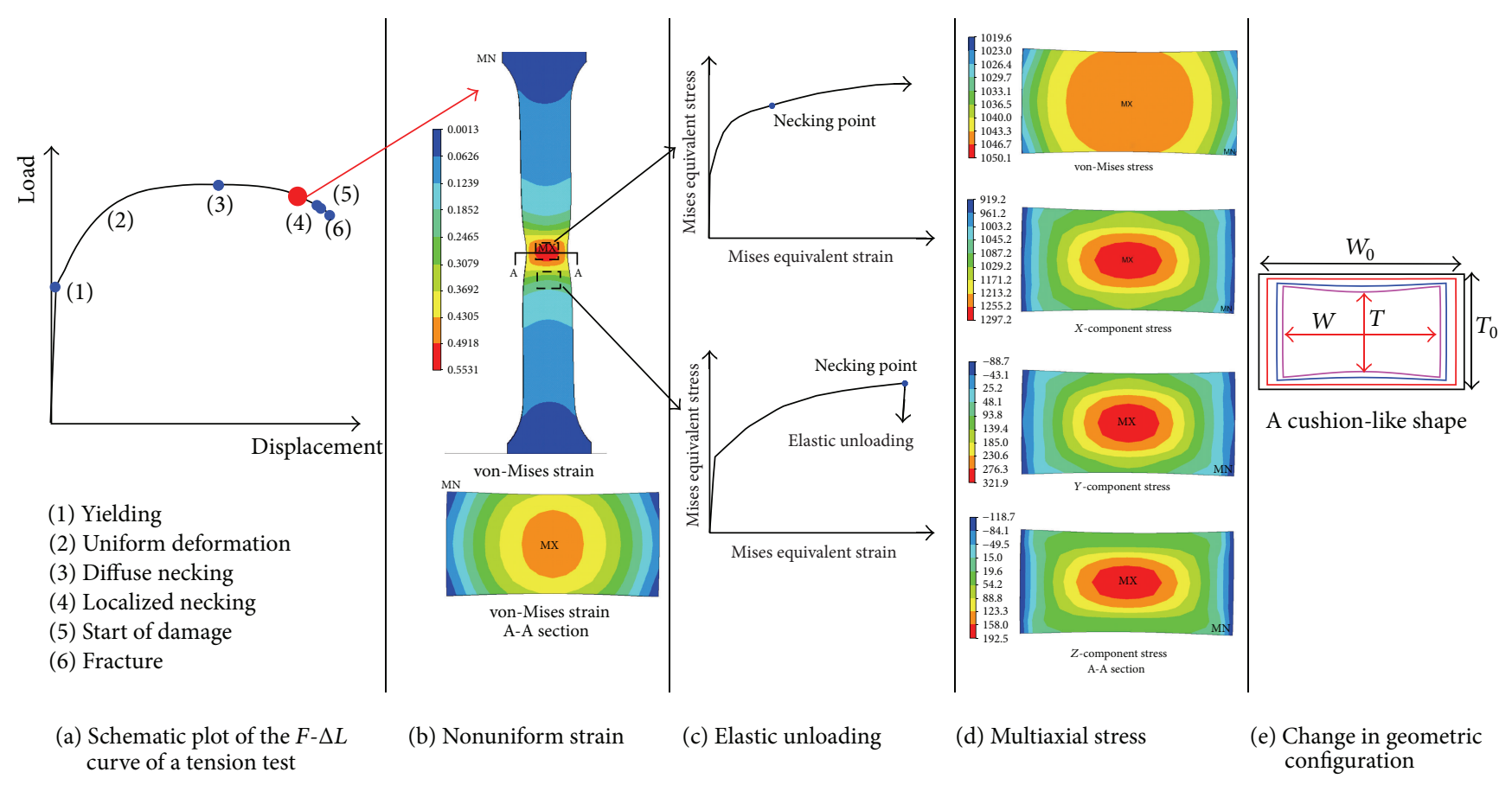

FIGURE 1: The difficulties in determining the true stress-strain curve at very large strains from uniaxial tensile test.

localized necking phenomenon of sheet specimens, where the plastic deformation is not diffused but rather is more concentrated and inclined at an angle to the tension axis [2,9]. Therefore, it is questionable to simply extrapolate the true constitutive relation of a ductile metal beyond the uniform elongation from the experimental measured data.

Bridgman developed some approximate analytical solutions to obtain the effective stress-strain curve of uniaxial tensile specimens beyond diffuse necking by utilizing various simplifications and assumptions about the necking geometry and stress and strain distribution inside the neck [10, 11]. In fact, his analytical approach provided a correction parameter which could transform the "average true axial stress" in the necking into the equivalent stress. However, a practical difficulty must be mentioned, as discussed in some detail by Ling [12] and Joshi et al. [13], that is, how to trace the true area of necking section and calculate the true strain during the tension test, which not only is a function of loading, but also depends on section's aspect ratio and material's plastic hardening. The traditional method (lateral extensometer measurement technique) is not up to this work, because the position of necking is random and very difficult to determine beforehand, particularly for the rectangular specimens whose cross section at the largest deformed zone forms a cushionlike shape [14] (see Figure 1(e)). Furthermore, Bridgman correction is not expected to be applicable to rectangular specimens, especially when localized necking is being developed [12], as it assumes an axisymmetric stress and strain field with constant plastic strain in the smallest necking section, while the strain gradients in the cross section of rectangular specimens increase with the increasing of aspect ratio [14].

In recent years, many research efforts have been carried out to develop a more accurate and reliable method to extract the true stress-strain curve beyond necking by utilizing finite element method (FEM). Cabezas and Celentano [3] used FEM to simulate the tensile deformation process and find correction factors for sheet specimens. Zhang et al. [2] proposed an empirical equation based on the experimental load-thickness curve to determine the true stress-strain curve of a rectangular specimen. Scheider et al. [14] found that the empirical method proposed by Zhang is not sufficiently accurate and the thickness reduction at the minimum section of the test coupon is difficult to measure; therefore he proposed instead the utilization of the experimental load-width curve. However, most conclusions of the above literatures were achieved by importing an assumed power law constitutive relation, which has been proved quite conservative and limited for some metals at large strains $[12,15,16]$. Zhano and Li [17] and Joun et al. [18] proposed an iterative approach to obtain the true stress-strain curve of a cylindrical sample up to fracture based on experimental $F-\Delta L$ curve and FEM simulations. However, their customized program is difficult to be implemented in a general commercial finite element program. Ling [12] analyzed the uniaxial tension of sheet specimens in the commercial program ABAQUS and proposed a weighted-average method to approximate the true stress by using the weighted average of the lower and upper bounds defined by power law and linear functions; then, a trial-and-error method was employed to determine the adjustable weight parameter. However, this method was based on an extrapolation of the stress-strain curve before necking and thus not suitable for calculations with high strains. Recently, a new experimental measurement tool of high resolution digital camera (DIC) was employed by some researchers $[14,15,19-21]$ to overcome the difficulties in tracing the change in the geometry of specimen. Dan et 
al. [19] used a digital camera and a coarse grid method to obtain the local strain field and then average surface axial true strain in steel sheet specimens; however their data were only applicable to moderate strains just after diffuse necking as no further correction due to multiaxial stress state was adopted. A similar method was also implemented in [20].

In the present study, for reasons of clarity of some fundamental definitions and concepts, the traditional analytical methods (TAM) are first reviewed and discussed briefly. Then, we introduce an experimental-numerical combined method (ENM) by using the simple uniaxial tension test and a general purpose commercial 3D finite element analysis (FEA) program to extract the true stress-strain curves after necking of metal coupons with round and rectangular geometries. This methodology utilizes an iterative scheme to minimize the errors between the simulated and experimental $F-\Delta L$ curves by modifying the imported stress-strain data step by step, and the true stress is determined when the error is less than an infinitesimal value. Seeing that some material damage (void nucleation and growth) will occur and accumulate gradually at the later stage of necking and the process from the onset of local necking to fracture [6], we focus on the nonuniform flow process before damage nucleation. No material failure and strain softening behavior are assumed in the analysis as the basic isotropic elastic-plastic constitutive model cannot predict the plastic deformation behavior when damage begins to accumulate. After describing the fundamentals of the present approach in detail, a MATLAB program is written to implement this method and verify the robustness and effectiveness of the proposed method. Compared with several material constitutive equations, the composite Line-Power Model and Misiolek equation are found to be very close to the FE-computed curves after necking. Finally, some suggestions are offered for possible improvements in both experimental measurements and FEA.

\section{Review of Traditional Analytical Methods (TAM)}

In simple tension tests, two well-defined stages are recognized. The first stage takes place before external load reaches the maximum value, and a homogeneous response is found. After the maximum load, the postplastic regime is characterized for a well-marked necking in the central zone of the test samples.

In the first stage of the process, the applied force $F$ and the elongation $\Delta L$ are measured and can be converted to the engineering stress $\sigma_{\mathrm{E}}$ and strain $\varepsilon_{\mathrm{E}}$ by

$$
\begin{gathered}
\varepsilon_{\mathrm{E}}=\frac{\Delta L}{L_{0}}, \\
\sigma_{\mathrm{E}}=\frac{F}{A_{0}},
\end{gathered}
$$

where $A_{0}$ and $L_{0}$ are the initial cross section and length of the specimen, respectively. These quantities are restricted to small uniform deformation. For large uniform deformation, the response is defined in terms of the axial strain $\varepsilon_{z}$ and axial stress $\sigma_{z}$ which coincides with the average axial stress $\left(\sigma_{z}\right)_{\mathrm{a}}$ :

$$
\begin{aligned}
& \varepsilon_{z}=\int_{L_{0}}^{L} \frac{d l}{l}=\ln \frac{L}{L_{0}}=\ln \left(1+\varepsilon_{\mathrm{E}}\right), \\
& \sigma_{z}=\left(\sigma_{z}\right)_{\mathrm{a}}=\frac{F}{A}=\sigma_{\mathrm{E}}\left(1+\varepsilon_{\mathrm{E}}\right),
\end{aligned}
$$

where $A$ and $L$ are the instantaneous cross section and length of the specimen, respectively. In this stage, axial stress and strain are coincident with the von-Mises equivalent stress $\bar{\sigma}$ and equivalent strain $\bar{\varepsilon}$, respectively.

Equations (2)-(3) are correct and applicable to both cylindrical and rectangular specimens as long as the elongation of tensile specimen is uniform. However, in the second stage of the process after the maximum load, necking occurs at the central zone of samples; deformation becomes nonuniform and the stress state triaxial. All the factors mentioned above have to be taken into account to obtain the true stress versus true strain. Due to complex stress-strain state and different assumptions adopted at the necking stage, the analytical methods for both cylindrical and rectangular specimens need to be discussed, respectively.

2.1. Analytical Method for Cylindrical Specimens. In developing an expression for the strain field beyond necking for a round specimen, Bridgman [10, 11] assumed that strain distributes uniformly in the minimum section with

$$
\varepsilon_{r}=\varepsilon_{\theta}=-\frac{\varepsilon_{z}}{2},
$$

where $\varepsilon_{r}, \varepsilon_{\theta}$, and $\varepsilon_{z}$ are the radial, hoop, and axial stress, respectively. Then it can be deduced that the equivalent strain $\bar{\varepsilon}$ at the minimum section is equal to the average axial strain. Introducing in (2) the volume conservation condition $A L=$ $A_{0} L_{0}$, then (2) is transformed into

$$
\bar{\varepsilon}=\varepsilon_{z}=\ln \frac{A_{0}}{A}=2 \ln \frac{D_{0}}{D},
$$

which means that, to obtain the equivalent strain at the neck, one only has to measure the instantaneous dimensions of the minimum section, that is, the current diameter $D$ of the necking section for round specimens.

The average axial stress $\left(\sigma_{z}\right)_{\mathrm{a}}$ at the smallest cross section can be calculated by (3). However, the radial and hoop stress, $\sigma_{r}$ and $\sigma_{\theta}$, are not equal to zero after necking; thus the average axial stress $\left(\sigma_{z}\right)_{\mathrm{a}}$ is not equal to the equivalent stress. Because the shear stresses disappear at the smallest part of the cross section, the equivalent stress $\bar{\sigma}$ under the assumption of a von-Mises yield condition is given by

$$
\begin{aligned}
\bar{\sigma} & =\sqrt{\frac{3}{2} \sigma_{i j}^{\prime} \sigma_{i j}^{\prime}} \\
& =\sqrt{\frac{1}{2}\left[\left(\sigma_{\mathrm{I}}-\sigma_{\mathrm{II}}\right)^{2}+\left(\sigma_{\mathrm{II}}-\sigma_{\mathrm{III}}\right)^{2}+\left(\sigma_{\mathrm{III}}-\sigma_{\mathrm{I}}\right)^{2}\right]},
\end{aligned}
$$

where $\sigma_{i j}^{\prime}$ represents the deviatoric stresses and $\sigma_{\mathrm{I}}, \sigma_{\mathrm{II}}$, and $\sigma_{\text {III }}$ refer to the principal stresses, respectively. For a round 


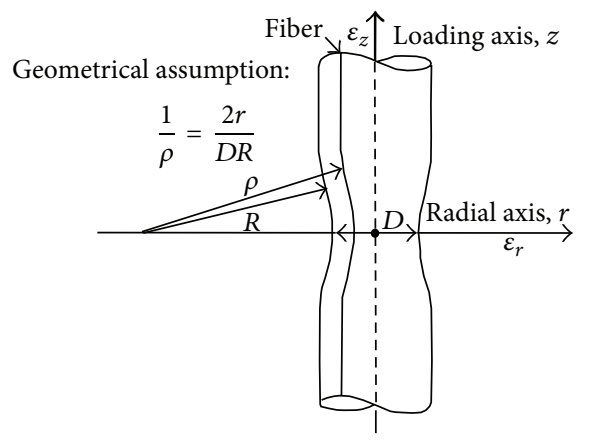

$\rho=$ radius of curvature of the grid line

$r=$ radius of actual cross section

$D=$ diameter of minimum section

$R=$ radius of curvature of the neck

FIGURE 2: Illustration of necking of a round specimen and introduction of parameters and geometrical assumption employed in this section.

bar, we have $\sigma_{\mathrm{I}}=\sigma_{z}$ and $\sigma_{\mathrm{II}}=\sigma_{\mathrm{III}}=\sigma_{r}=\sigma_{\theta}$. Based on the geometrical assumption shown in Figure 2 and stress equilibrium equations at the minimum section, Bridgman $[10,11]$ gave the stress distribution formulas at the necking section:

$$
\begin{aligned}
& \sigma_{r}(r)=\sigma_{\theta}(r) \\
& =\frac{\left(\sigma_{z}\right)_{\mathrm{a}}}{(1+4 R / D)}\left[\frac{\ln \left(\left(D^{2} / 4+D R-r^{2}\right) / D R\right)}{\ln (1+D / 4 R)}\right], \\
& \sigma_{z}(r) \\
& =\frac{\left(\sigma_{z}\right)_{\mathrm{a}}}{(1+4 R / D)}\left[\frac{1+\ln \left(\left(D^{2} / 4+D R-r^{2}\right) / D R\right)}{\ln (1+D / 4 R)}\right] .
\end{aligned}
$$

Then the maximum equivalent stress $\bar{\sigma}$ at the center of the specimen (i.e., $r=0$ ) can be calculated from (6) by substituting (7):

$$
\begin{aligned}
\bar{\sigma} & =\frac{\left(\sigma_{z}\right)_{\mathrm{a}}}{(1+4 R / D) \ln (1+D / 4 R)}=C_{\mathrm{ro}}\left(\sigma_{z}\right)_{\mathrm{a}}=\frac{F}{A} C_{\mathrm{ro}}, \\
C_{\mathrm{ro}} & =\left[\left(1+\frac{4 R}{D}\right) \ln \left(1+\frac{D}{4 R}\right)\right]^{-1},
\end{aligned}
$$

where $C_{\mathrm{ro}}$ may be considered as a correction factor which transforms the average axial stress in the necking into the equivalent stress.

Verification of this correction method is difficult because true stress at the minimum section after necking cannot be measured directly. Many finite element analyses have shown that stress distribution at the minimum section approximately follows (7), and the strain distribution also mainly exhibits uniform feature along the necked section which is consistent with the basic assumption. However, it must be pointed out that Bridgman correction method is not convenient to use in practice because it requires a series of tests with different loadings to determine the radius of curvature $R$ and the minimum diameter $D$ which are both difficult to measure with sufficient accuracy. In order to avoid the measurement of $R$, Bridgman [10] suggested the simplification

$$
\frac{D}{R}=2 \sqrt{\varepsilon_{z}-0.1} \quad\left(\varepsilon_{z}>0.1\right) .
$$

Then (9) can be further simplified to

$$
\begin{aligned}
C_{\text {ro }}=\left[\left(1+\frac{2}{\sqrt{\varepsilon_{z}-0.1}}\right) \ln \left(1+\frac{\sqrt{\varepsilon_{z}-0.1}}{2}\right)\right]^{-1} \\
\left(\varepsilon_{z}>0.1\right) .
\end{aligned}
$$

2.2. Analytical Method for Rectangular Specimens. Early research carried out by Zhang et al. [2] stated that for thin sections it is better to use test specimens with a rectangular section to determine the constitutive relation. Based on the same assumptions mentioned above for cylindrical specimens, Bridgman extended his correction method to rectangular specimens. However, the necking of flat sample has been proved to be much more complicated than that of round bar. The strain distribution at the minimum cross section becomes highly nonuniform after necking and the strain gradients in the cross section of rectangular specimens increase with the increasing of aspect ratio [14]. Moreover, the previous experimental works conducted by Aronofsky [22] suggested that the geometrical assumption employed in Bridgman formula did not apply to rectangular specimens. Therefore, it is not surprising that the Bridgman correction formulas apparently have not been applied in flat specimens successfully. Some other attempts to determine the true stress-strain relation with rectangular specimens have been proposed in literatures $[2,3,12,14,18]$, among which the Scheider procedure [14] is commonly used and thus is discussed and reviewed here.

After the onset of necking, the actual area of the necking section cannot be measured or calculated due to the "cushion-like shape"; see Figure 1. Thus, the average axial stress $\left(\sigma_{z}\right)_{\mathrm{a}}$ cannot be determined by employing (3). A nominal area needs to be defined:

$$
\widetilde{A}=b^{2} \frac{t_{0}}{b_{0}},
$$

where $t_{0}$ and $b_{0}$ are the initial thickness and width of flat specimen, respectively, and $b$ represents the instantaneous width of necking section. The idea of relating the true effective stress to the measured load, that is, correction method, can still be adopted:

$$
\bar{\sigma}=\frac{F}{\widetilde{A}} C_{\text {re }} .
$$

Based on a series of numerical simulations, an empirical formula to calculate the correction factor $C_{\mathrm{re}}$ of rectangular specimens after necking was developed by Scheider [14]:

$$
C_{\mathrm{re}}= \begin{cases}1 & \bar{\varepsilon}<1.42 \bar{\varepsilon}_{u} \\ 0.22\left(\bar{\varepsilon}-1.42 \bar{\varepsilon}_{u}\right)(\bar{\varepsilon}-0.78)+1 & \bar{\varepsilon}>1.42 \bar{\varepsilon}_{u},\end{cases}
$$


where $\bar{\varepsilon}_{u}$ is the limit value of uniform strain, that is, the equivalent strain at maximum load. Note that, different from the Bridgman correction factor $C_{\mathrm{ro}}$, the correction factor $C_{\text {re }}$ for rectangular specimens includes not only the effect of multiaxial stress state but also the transition from the nominal area $\widetilde{A}$ to the actual area $A$. In (14), $\bar{\varepsilon}$ represents the total equivalent strain, which can be written as the sum of elastic strain $\bar{\varepsilon}^{\mathrm{e}}$ and plastic strain $\bar{\varepsilon}^{\mathrm{p}}$; that is, $\bar{\varepsilon}=\bar{\varepsilon}^{\mathrm{e}}+\bar{\varepsilon}^{\mathrm{p}}$, with

$$
\bar{\varepsilon}^{\mathrm{p}}=\int_{0}^{t} \dot{\bar{\varepsilon}}^{\mathrm{p}} d t=\int_{0}^{t} \sqrt{\frac{2}{3} \dot{\varepsilon}_{i j}^{\prime \mathrm{p}} \dot{\varepsilon}_{i j}^{\prime \mathrm{p}}} d t .
$$

Because of the condition of incompressibility, $\dot{\varepsilon}_{\mathrm{I}}^{\mathrm{p}}+\dot{\varepsilon}_{\mathrm{II}}^{\mathrm{p}}+\dot{\varepsilon}_{\mathrm{III}}^{\mathrm{p}}=0$, (15) reduces to

$$
\bar{\varepsilon}^{\mathrm{p}}=\int_{0}^{t} \sqrt{\frac{4}{3}\left(\left(\dot{\varepsilon}_{\mathrm{I}}^{\mathrm{p}}\right)^{2}+\dot{\varepsilon}_{\mathrm{I}}^{\mathrm{p}} \dot{\varepsilon}_{\mathrm{II}}^{\mathrm{p}}+\left(\dot{\varepsilon}_{\mathrm{II}}^{\mathrm{p}}\right)^{2}\right)} d t .
$$

Since elastic strain is relatively small compared to plastic strain at large deformation regime, the contribution of elastic strain can be neglected. In most cases, due to the nonlinearity of the deformation, the integration results of (16) will be inconsistent with the calculated equivalent strain using the total deformation and yield the following expression:

$$
\bar{\varepsilon} \geq \sqrt{\frac{4}{3}\left(\varepsilon_{\mathrm{I}}^{2}+\varepsilon_{\mathrm{I}} \varepsilon_{\mathrm{II}}+\varepsilon_{\mathrm{II}}^{2}\right)},
$$

where the local strains $\varepsilon_{\mathrm{I}}$ and $\varepsilon_{\mathrm{II}}$ are measured directly on the specimen surface at the necking section:

$$
\begin{gathered}
\varepsilon_{\mathrm{I}}=\ln \left(1+\frac{\partial u_{x}}{\partial X}\right), \\
\varepsilon_{\mathrm{II}}=\ln \left(1+\frac{\partial u_{y}}{\partial Y}\right) .
\end{gathered}
$$

In (18), $X$ and $Y$ are the reference coordinates in length and width direction of the specimen, respectively. Note that only in the special cases, that is, when the principal strain directions remain unchanged and the local stresses increase monotonically, does the equality sign hold in (17). However, a series of numerical studies conducted by Scheider [14] have additionally shown that the error induced by the application of (17) is far less than that induced by the summation of increments when the load steps of FEM are relatively large. Thus, in this paper, we can still approximately calculate the equivalent strain $\bar{\varepsilon}$, without significant loss of accuracy, by employing (17) with the equality sign.

In this paper, we use (2), (3), (5), (8), and (11) to calculate the true stress-strain curve of cylindrical specimens, and for rectangular specimens, (2), (3), (13), (14), and (17) can be used.

\section{Proposed Experimental-Numerical Combined Method (ENM)}

Here we propose a new experiment-numerical combined method (ENM) to obtain the true stress-strain curve of a ductile metal after necking by tracing the experimental $F-\Delta L$ curve using numerical simulations. According to ENM, the experimental $F-\Delta L$ curve is considered as a target, the simulated $F-\Delta L$ curve is forced to approach the target step by step by modifying the imported true stress-strain data in FEM software and performing iterative computations, and then the true stress-strain data will be determined when the error between experimental and simulated $F$ is less than a given limit value. Therefore, a precision experimental $F-\Delta L$ curve obtained by the tensile test is the most important prerequisite to ensure the accuracy of ENM. Before conducting iterative computations, the true stress-strain data of samples before necking should be first calculated by (2)-(3) as the initial data of finite element iteration. It has been revealed that the necking point can be exactly predicted with these data [1]. Then an iterative algorithm based on the above idea is adopted, which is written as follows.

Step 1. Divide the stress-strain curve before necking into a certain number of smaller stages to approximate the real curve according to the required precision. Define an arithmetic series as the "strain point" $\operatorname{strain}^{i}(i=1,2, \ldots, M)$ needs to be iterated according to a given interval $(\Delta)$ :

$$
\operatorname{strain}^{i}=\varepsilon_{0}+i \Delta \quad(i=1,2, \ldots, M),
$$

where $\varepsilon_{0}$ represents the end of the uniform stress-strain curve which can be used to describe the formation of necking.

Step 2. Define stress $1^{i}$ and stress $2^{i}$ (corresponding to Line 1 and Line 2 in Figure 3(a)) at strain ${ }^{i}$. Note that Line 1 and Line 2 are the horizontal line and tangent line at strain ${ }^{i}$, respectively.

Step 3. Let stress ${ }^{i}=$ stress $^{i}$. stress $^{i}$ represents the assumed stress value corresponding to strain ${ }^{i}$ on the stress-strain curve after necking.

Step 4. Import the stress-strain data less than or equal to strain $^{i}$ and make computations. Then extract the simulated load $\left(P^{i}\right)$, simulated displacement $\left(\delta^{i}\right)$, and experimental load $\left(F^{i}\right)$ corresponding to strain ${ }^{i}$. The linear interpolation method is utilized if the data output from FEA do not coincide exactly with the experimental measured data.

Step 5. Check the convergence of the solution at strain ${ }^{i}$ by comparing $P^{i}$ with $F^{i}$ and calculate Error ${ }^{i}$ by

$$
\text { Error }^{i}=\left|\frac{\left(F^{i}-P^{i}\right)}{F^{i}}\right| .
$$

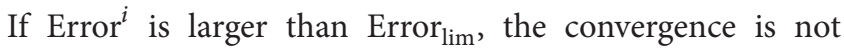
achieved, and the following algorithms are used to update stress $^{i}$ :

$$
\begin{aligned}
& \text { if } P^{i}>F^{i}, \\
& \text { then, stress } 1^{i}=\text { stress }^{i}, \\
& \text { stress }^{i}=(1-a) \times \operatorname{stress}^{i}+a \times \operatorname{stress}^{i},
\end{aligned}
$$






(a)

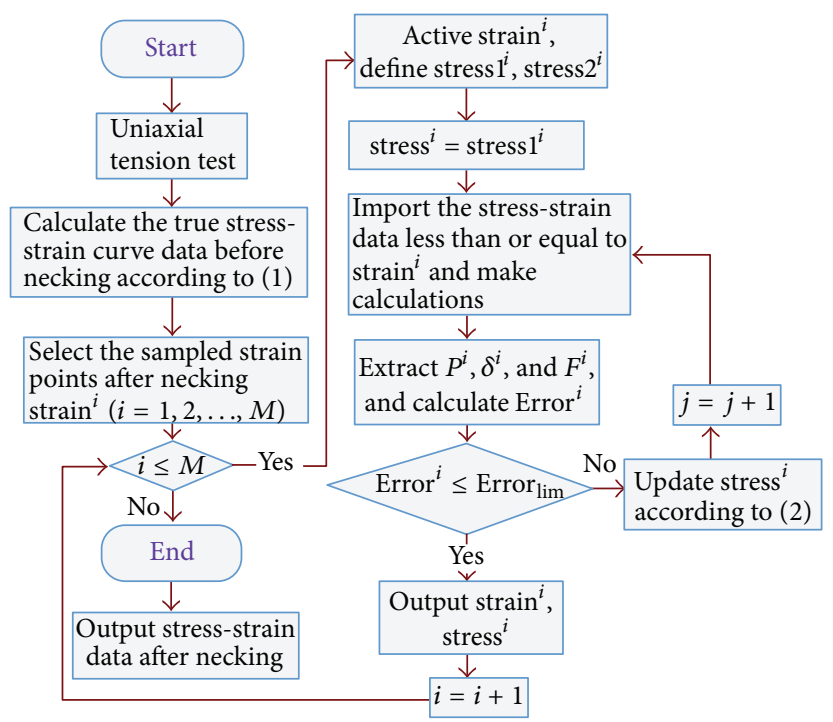

(b)

FIgURE 3: (a) The modification processes of the true stress at "active strain point" $\operatorname{strain}^{i=1}$. (b) The flow chart of ENM.

$$
\begin{aligned}
& \text { if } P^{i}<F^{i} \text {, } \\
& \text { then, stress } 2^{i}=\text { stress }^{i} \text {, }
\end{aligned}
$$

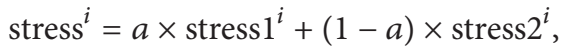

where $a=1 / n$, where $n$ is a parameter controlling the convergence rate whose effect will be discussed in the next section. Error ${ }_{\text {lim }}$ is a subjective parameter which can be determined according to the actual needs ( $0.1 \%$ is adequate). Obviously, when Error $_{\text {lim }}$ takes a relatively small value, more computation time will be spent on the iterations and more accurate results will be determined. If the convergence is achieved, stop the iterations, replace $i$ with $i+1$, and return to Step 2. This iterative procedure and subsequent stress update can continue in the same way until all stress-strain data are determined.

To facilitate understanding the basic principle of ENM, Figure 3(a) takes the "active strain point" $\operatorname{strain}^{i}$ as an example to illustrate the iterative procedures. The detailed procedure of proposed method is summarized in Figure 3(b). According to the above elaborations and the flow chart in Figure 3(b), we write a special MATLAB program to automatically implement this algorithm. The numerical computation is carried out by using ANSYS for its powerful parametric design language (APDL). The information which needs to be input into the program includes the following:

(a) Sample dimensions and finite element mesh information (element type and density).

(b) Material properties: elastic modulus, Poisson's ratio, and true stress-strain curve data before necking. (c) Load information.

(d) Iterative information: the value of "strain point" strain $^{i}$ and error limits.

(e) Experimental $F-\Delta L$ curve.

Note that, in order to save the computation time, we employ the method of Restart Analysis in FEM. The computation is terminated when the maximum von-Mises strain in the necking section is greater than strain ${ }^{i}$, and the subsequent iterations will start from the interrupted point of previous step. Experience has proved that this method can save nearly $60 \%$ of the computation time.

\section{Application and Validation}

The main objective of the present analysis is to validate the predictions of ENM with the experimental data in [3] in order to achieve an adequate mechanical characterization when cylindrical and rectangular specimens are both considered. The chosen material is SAE 1045 steel and the average chemical composition is shown in Table 1. The employed geometric configurations for test specimens are sketched in Figure 4. The average experimental measured values for the yield strength, maximum load, maximum engineering stress, and elongation at the fracture stage are summarized in Table 2. F- $\Delta L$ curve and dimensions of necking cross section are measured simultaneously during the loading process. Further details about the test procedure can be found in [3].

An isotropic elastic-plastic material model is used in the nonlinear FEA of the tension test with the commercial FE program ANSYS. The nonlinear strain hardening of the plastic deformation of each specimen is characterized by the von-Mises effective stress-strain curve. A set of discrete data of effective stress and strain are used as the input in each 
TABLE 1: Chemical composition of SAE 1045 (wt\%) [3].

\begin{tabular}{lcccccccc}
\hline $\mathrm{C}$ & $\mathrm{Si}$ & $\mathrm{Mn}$ & $\mathrm{P}$ & $\mathrm{S}$ & $\mathrm{Cr}$ & $\mathrm{Ni}$ & $\mathrm{Al}$ & $\mathrm{Cu}$ \\
\hline 0.447 & 0.213 & 0.756 & 0.0148 & 0.033 & 0.064 & 0.091 & 0.0011 & 0.277
\end{tabular}

TABLE 2: Mechanical properties of SAE 1045 steel [3].

\begin{tabular}{lcccc}
\hline Sample & $f_{y}(\mathrm{MPa})$ & $P_{u}(\mathrm{kN})$ & $f_{u}(\mathrm{MPa})$ & $\delta(\%)$ \\
\hline Cylindrical & 450.4 & 46.6 & 749.2 & 18.5 \\
Rectangular & 451.6 & 56.5 & 762.0 & 20.0 \\
\hline
\end{tabular}

$f_{y}$ : yield strength, $P_{u}$ : maximum load, $f_{u}$ : maximum engineering stress, and $\delta$ : elongation at fracture.

FEA through a table. To overcome the convergence difficulty caused by the large variation of the derived work-hardening rate, especially just before and after necking, no fewer than 30 data points are selected to ensure a smooth change of work-hardening rate. Owing to the isotropic symmetry, only one-eighth of the specimen is modeled here to reduce computation time. The mesh of the FE model of the tensile specimen is shown in Figure 4. A refined mesh is applied at the center of each coupon where necking is expected to occur. Meshes with 200 elements in the necking area are employed for the objectives of the work. The convergence of computed results in terms of mesh density has been checked to be successfully reached at this level. Note that in the experiment necking occurs at a point caused by a preset imperfection at the center of the specimen, while in the simulation the mesh is made without imperfections and the specimen necks at the corresponding edge of the mesh. The load is applied by controlling displacement and a set of specific boundary conditions are imposed to satisfy the symmetry conditions. No strain rate effect is considered. To check the robustness of ENM, different values of parameter $n(5,10$, and 20) in Step 5 are employed in the iterative procedure.

Figure 5 shows the comparison of true stress-strain curves determined from TAM and ENM with different values of parameter $n$ by applying to cylindrical and rectangular specimens. As can be seen, parameter $n$ has little effect on the iterative results, although different computation time will be consumed when $n$ takes different values. Experience has shown that it can probably achieve a balance between the precision and computation time when parameter $n$ equals 10 . In order to verify the accuracy of the proposed ENM procedure, the obtained material true constitutive relations shown in Figure 5 are applied to tensile simulations, and then we can extract the information on load and displacement from the numerical results during loading procedure. Figure 6 shows the comparisons between the simulated and experimental $F-\Delta L$ curves of both cylindrical and rectangular specimens. It can be seen that, for these two specimens, the material true constitutive relation obtained by ENM is in good agreement with the experimental results in large deformation analysis; however, the simulated $F-\Delta L$ curves based on TAM present a conservative tendency after necking. An additional FEA with a much denser mesh (up to 4 times more elements) is also employed to confirm the convergence of the computed stressstrain and the representative results for the two specimens

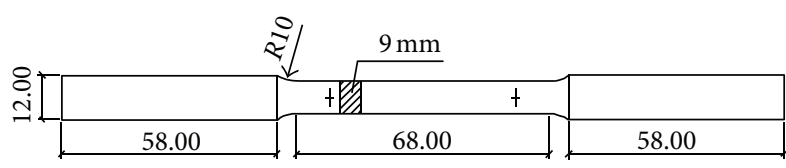

(a)

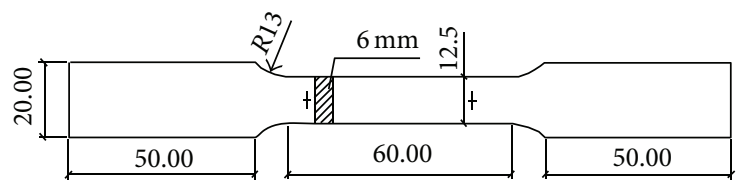

(b)

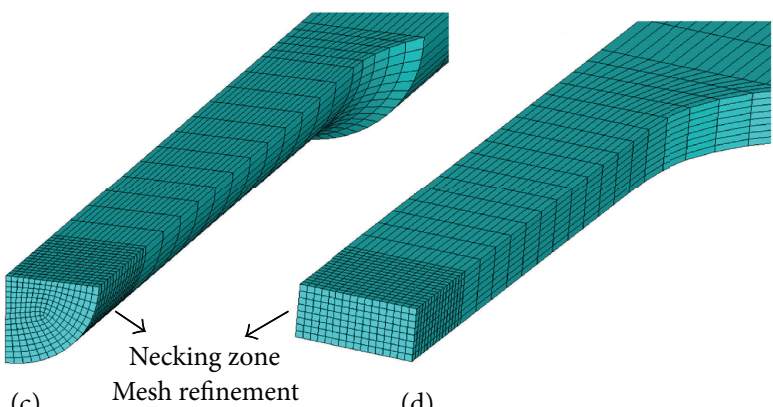

(c) Mesh refinement

(d)

FIgURE 4: Geometric configurations for (a) cylindrical and (b) rectangular samples and finite element mesh for (c) cylindrical and (d) rectangular specimens.

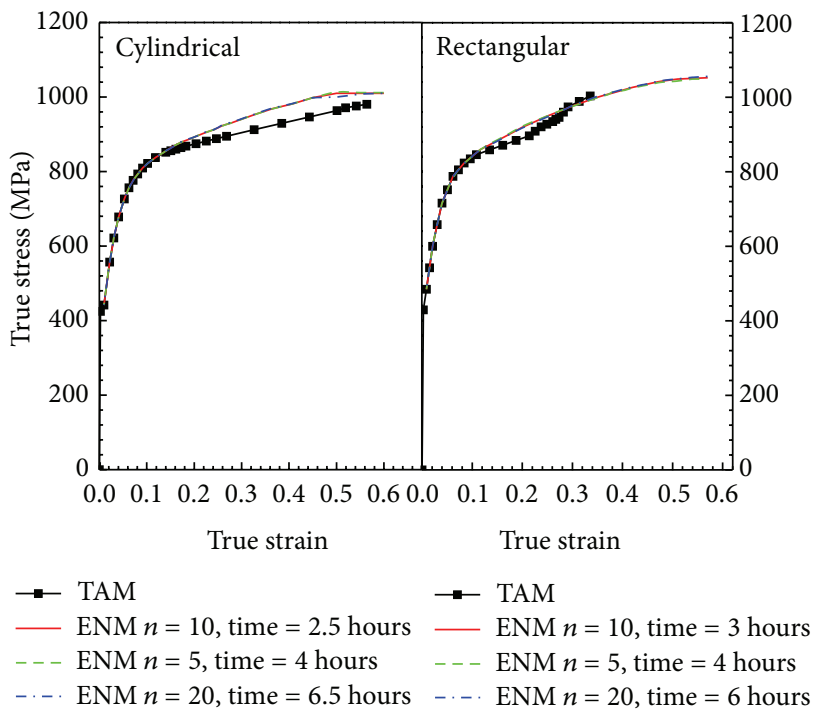

FIGURE 5: True stress-strain curves determined from TAM and ENM $(n=5,10$, and 20).

are shown in Figure 6: little deference is observed between the FE-computed $F-\Delta L$ curves, and they both closely match the experimental curves. This fully demonstrates that the convergence of computed results in terms of mesh density can be successfully reached at the level indicated in Figure 4.

Besides the $F-\Delta L$ curves, further details of necking zone such as contours and dimensions can also be extracted from the numerical results. Figures $7(a)$ and $7(b)$ show some 


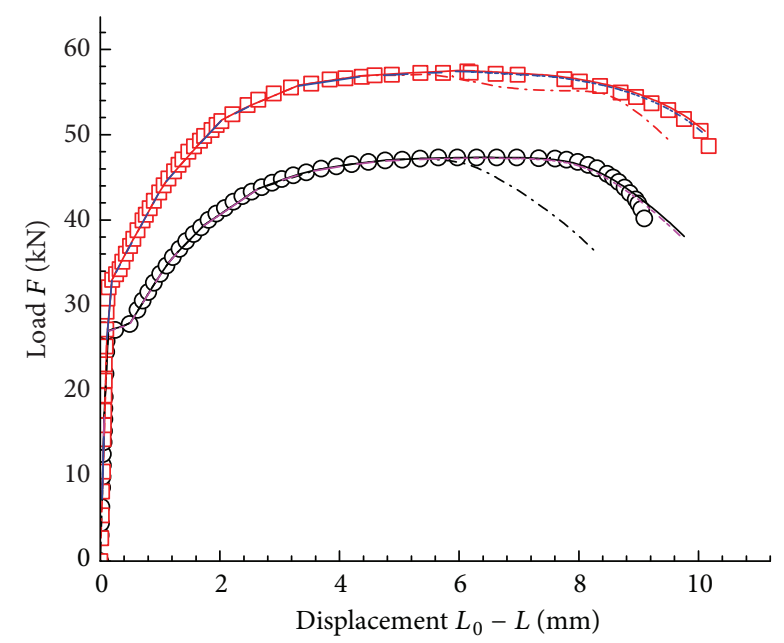

Cylindrical

- Experimental

— Simulated: ENM (200 elements)

-.. Simulated: TAM

— Simulated: ENM (800 elements)

Rectangular

- Experimental

— Simulated: ENM (200 elements)

-.-. Simulated: TAM

— Simulated: ENM (800 elements)

FIGURE 6: Experimental measured and computed $F-\Delta L$ curves.

detailed results at the cross section which undergoes extreme necking: ratio of current to initial transversal dimensions versus the elongation in the necking zone together with the mean true axial stress against the true strain. An overall consistent trend can be observed for both cylindrical and sheet specimens. The simulated load decreases after necking; however the simulated mean true axial stress (see Figure $7(\mathrm{~b}))$ continues increasing until fracture, consistent with the experimental results. It fully demonstrates that TAM and ENM can both capture the fact that a geometrical instability occurs and the area reduction predominates over the material hardening at the necking zone. The numerical prediction for the ratio of current to initial transversal dimensions in terms of the elongation starts with a linear relationship, reflecting uniform distributions of stress and strain. Afterwards, a sudden reduction of the transversal dimensions takes place causing the necking formation and the nonhomogeneous stress and strain distributions along the specimen. As can be seen from Figure 7(a), the inflection points, that is, necking points, obtained by ENM, appear at the elongation of $15 \%$ for round specimen and $12.5 \%$ for sheet specimen, respectively, which shows good consistence with the test results; however, for TAM, premature necking (at the elongation of 10\%) and serious deviation of the deformation curves after necking can be observed. Figure 7(c) compares the correction factor calculated by TAM with that obtained through the numerical simulation as the quotient between the maximum von-Mises equivalent stress at the neck and the mean axial true stress.
It can be seen that the numerical values of correction factor $C_{\text {ro }}$ for cylindrical specimen are significantly larger than that obtained from (11). The similar results were also found in the previous work conducted by Cabezas and Celentano [3] and García-Garino et al. [23]. It is precisely because of this reason that the simulated $F-\Delta L$ curve based on TAM is not in agreement with the experimental results after necking (see Figure 6). In this paper, in order to avoid measuring the ratio of necking curvature $R$, the simplified method of (11) is adopted in TAM. Thus, this also indicates that the simplified method of (11) cannot achieve the required accuracy or sufficient versatility in some circumstances. The assumptions and simplifications adopted in TAM are conducive for the accumulation of systematic errors and culminating in inaccurate results. On the other hand, for rectangular specimens, the range of stress correction factor $C_{\text {re }}$ obtained by TAM is very limited; consequently, the maximum strain value of the calculated constitutive relation after necking only reaches 0.4 , as shown in Figure 5. In addition, TAM requires that a series of data, such as the dimensions of necking section and radium of curvature of the neck, must be measured during the test; this will lead to restrictions in practical applications.

The above analysis shows that the true constitutive relation of a ductile metal can be conveniently obtained from the uniaxial tension test by employing the proposed ENM; furthermore, the numerical results also indicate that ENM can achieve a more accurate description of the mechanical response of the materials after necking formation than TAM. However, some slight "strain softening" is observed on the FE-computed effective stress-strain curves in Figure 5. Although some material damage (void nucleation and growth) may occur during the later stage of necking, the major factor contributing to the softening behavior is the limited "resolution" of the average strain measured by extensometer. Figure 8 plots the average axial strains of each transversal section of rectangular specimen along the gauge length at different displacements. The final effective stress-strain data in Figure 5 is used in the FEA. It can be seen that, before the displacement of $4.81 \mathrm{~mm}$, strain distribution in the flat part of the specimen is quite uniform, while a sharp strain subsequently appears when the necking occurs. The plastic deformation becomes so localized and inhomogeneous during the necking stage, while only a small portion of the material experiences the extreme plastic strain. Thus, the displacement over the whole gauge length will become much less sensitive to the plastic details inside the neck with the increasing of gauge length (see the dash line in Figure 8). Since the displacement data outside the neck region become less useful anyway for characterizing the material deformation behavior at large strains, a specimen with a shortest possible gauge length is thus preferred to be used in the experimental measurements. The DIC techniques will be tentatively introduced to extract the local surface strain at the necking region when a high accurate true stress-strain curve is required. Then the average surface axial strain and load data can be employed as the targets in the ENM, instead of relying on the global $F-\Delta L$ curve data. Another critical issue that should be mentioned is that DIC technology can only extract the distribution of surface strain 


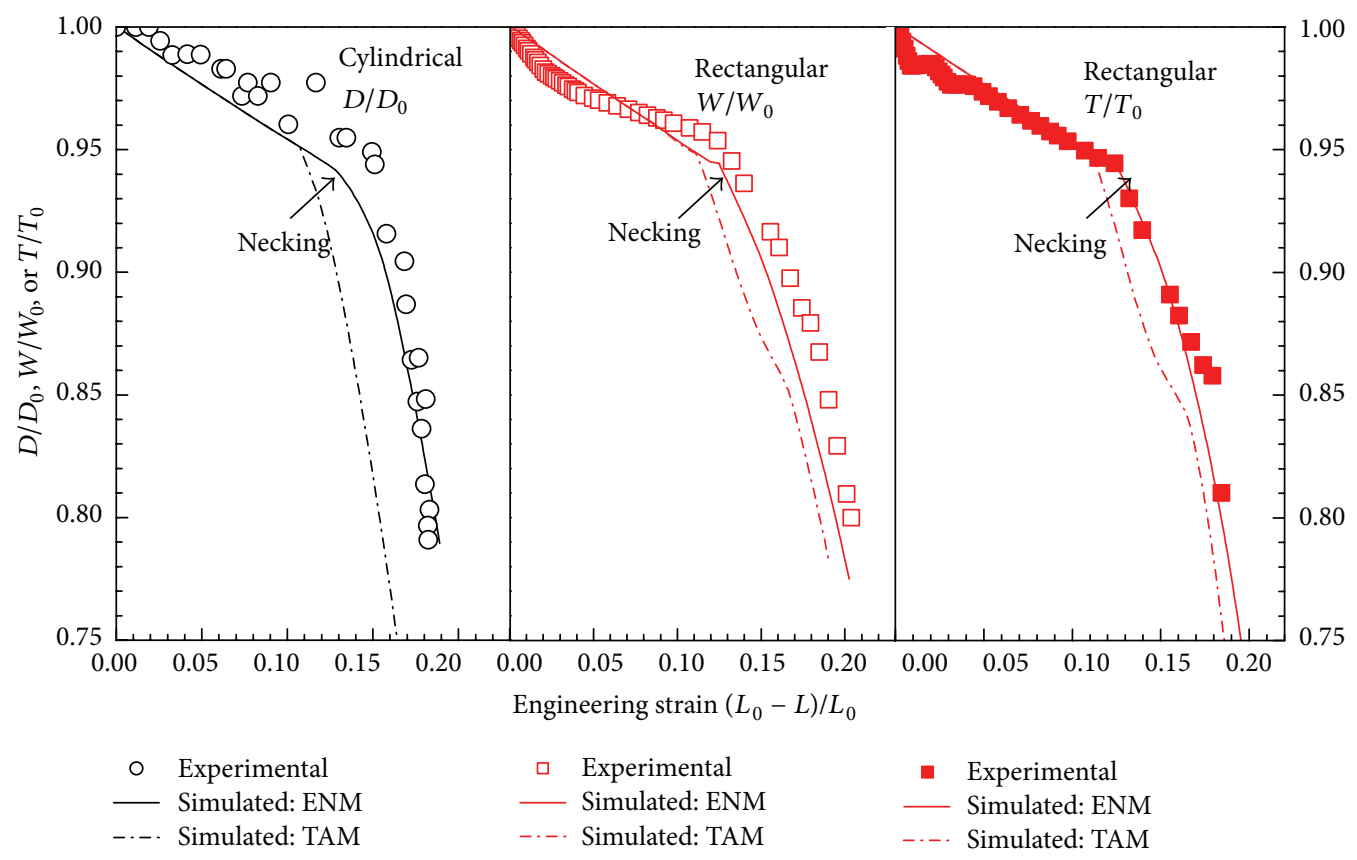

(a)

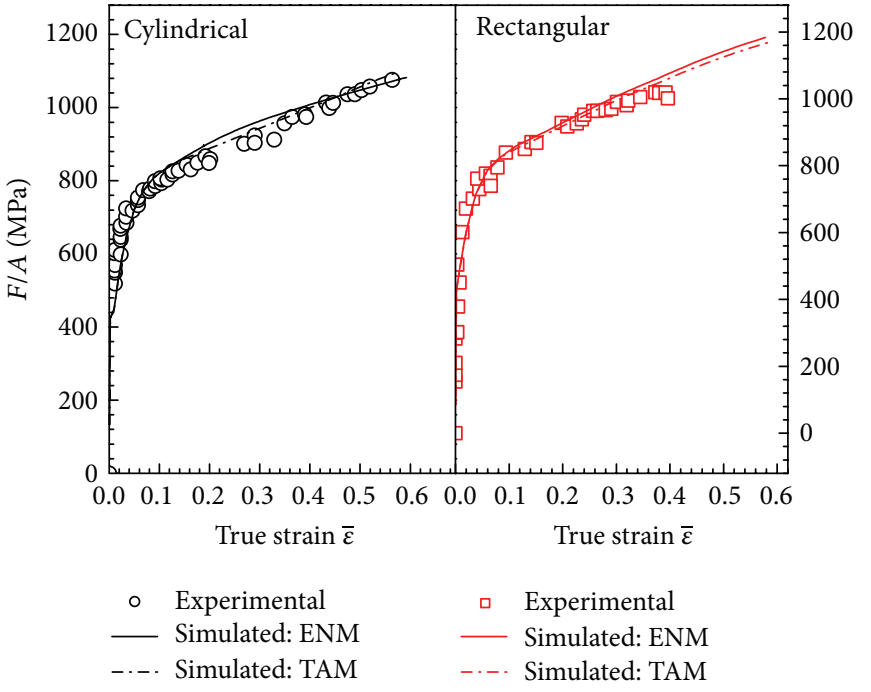

(b)

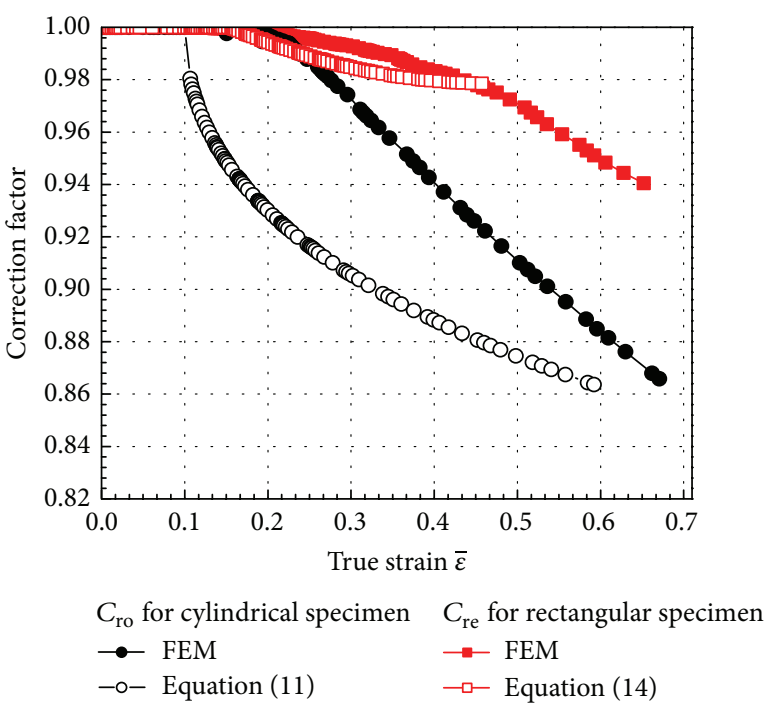

(c)

Figure 7: (a) Ratio of current to initial transversal dimensions versus axial elongation. (b) Mean true axial stress versus true strain. (c) Correction factors.

without considering the influence of inner damage evolution in the later stage of necking; therefore, in common with the traditional extensometer method, it cannot be used to obtain the material constitutive relationship at the later stage of necking when the damage begins to nucleate and accumulate. Researches on this aspect will be continuously carried out in our follow-up work. In most cases, the $F-\Delta L$ curve measured by extensometer can still satisfy the accuracy requests and is easy to implement.

Once the final discrete data points of true stress-strain curve are determined, we can carry out a curve-fitting routine to find the appropriate material parameters. Several experiential hardening laws have been used to fit the stressstrain curves before and after diffuse necking (see Figure 9), and the material parameters of these hardening laws are listed in Table 3. Note that the diffuse necking occurs under uniaxial tension per the Considére condition, expressed as

$$
\begin{gathered}
\left.\sigma\right|_{\varepsilon=\varepsilon_{u}}=\sigma_{u}, \\
\left.\frac{d \sigma}{d \varepsilon}\right|_{\varepsilon=\varepsilon_{u}}=\sigma_{u},
\end{gathered}
$$

where $\sigma_{u}$ and $\varepsilon_{u}$ refer to the true stress and true strain at the onset of diffuse necking. The fitting curves in Figure 9(a) 
TABLE 3: Material parameters for experiential hardening laws.

\begin{tabular}{|c|c|c|c|}
\hline \multirow{2}{*}{ Stage } & \multirow{2}{*}{ Equation name } & \multicolumn{2}{|c|}{ Material parameters } \\
\hline & & Cylindrical & Rectangular \\
\hline \multirow{3}{*}{ Before necking } & $\begin{array}{l}\text { (1) Hollomon } \\
\sigma=C \varepsilon^{n}\end{array}$ & $C=1078.6, n=0.1185$ & $C=1103, n=0.11577$ \\
\hline & $\begin{array}{l}\text { (2) Swift } \\
\sigma=C\left(\varepsilon-\varepsilon_{0}\right)^{n}\end{array}$ & $C=1066.7, \varepsilon_{0}=0.00947, n=0.10903$ & $C=1092.5, \varepsilon_{0}=0.00807, n=0.1077$ \\
\hline & $\begin{array}{l}\text { (3) Misiolek } \\
\sigma=C \varepsilon^{n} \exp \left(n_{1} \varepsilon\right)\end{array}$ & $C=2147.7, n=0.33836, n_{1}=-1.855$ & $C=1852.3, n=0.28, n_{1}=-1.4186$ \\
\hline \multirow{2}{*}{ After necking } & $\begin{array}{l}\text { (4) Misiolek } \\
\sigma=C \varepsilon^{n} \exp \left(n_{1} \varepsilon\right)\end{array}$ & $C=1034.6, n=0.1052, n_{1}=0.1122$ & $C=1031.2, n=0.09444, n_{1}=0.1846$ \\
\hline & $\begin{array}{l}\text { (5) Line-Power Model } \\
\sigma=w\left(n_{1} \varepsilon+n_{2}\right)+(1-w) C \varepsilon^{n}\end{array}$ & $\begin{array}{c}n_{1}=837.7, n_{2}=738.4, C=1078.6 \\
n=0.1185, w=0.1530\end{array}$ & $\begin{array}{c}n_{1}=859.4, n_{2}=760, C=1103 \\
n=0.11577, w=0.2343\end{array}$ \\
\hline
\end{tabular}

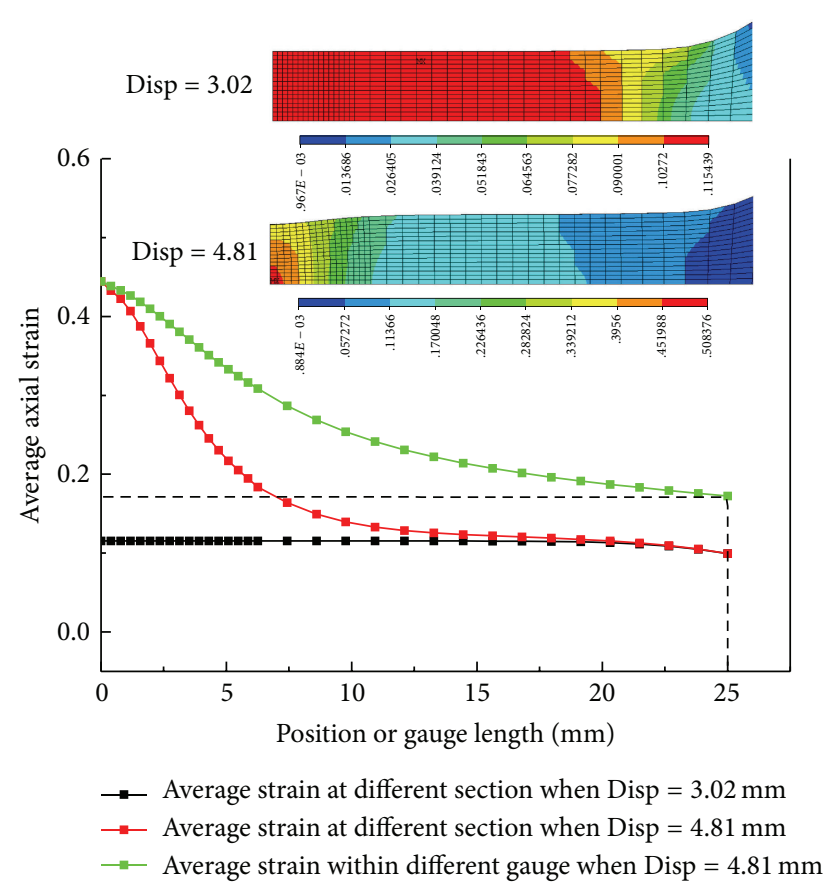

FIGURE 8: The average axial strain profile at different deformation stages and the average axial strain within different gauge length.

reveal that Misiolek equation can achieve a more accurate depiction of the work-hardening performance of the tested material before diffuse necking, which is consistent with the conclusion of [19]. The linear relationship and power law are, respectively, employed to fit the true stress-strain curve after necking. However, the power law extrapolation appears to underestimate the true stress, and the linear relationship generally leads to the overestimation of true stress (see Figure 9(b)). Thus, a composite model (Line-Power Model) [12] by combining the two simple models given above and setting an additional parameter (weight parameter $w$ ) is constructed to better describe the large strain behaviors and the fitted values of the single adjustable weight parameter $w$ are listed in Table 3. Figure 9(b) also demonstrates that the deformation behavior of the tested material after diffuse necking can also be depicted by Misiolek equation, which is essentially coincident with LP model. Note that the strain points larger than 0.5 are eliminated during the fitting process due to more or less softening behavior. The inset of Figure 9, as well as the parameters of hardening laws in Table 3, further indicates that the true stress-strain curve is an intrinsic characteristic of the material and not dependent on any geometric and dimensional parameters.

\section{Conclusions}

It is well-known that the stress-strain data obtained by a simple tension test using the conventional extensometer technique will underestimate the effective stress of ductile metal beyond necking. In this paper, a hybrid experimental and numerical method using the measured $F-\Delta L$ curve as the target for FE computations is proposed to obtain the material true constitutive relation after necking. A program-controlled iterative procedure is applied to successively modify the effective stress-strain curve and minimize the errors between the simulated and experimental $F-\Delta L$ curves. Compared with some simple correction methods, that is, TAM, the iterative procedure using FEA of a tensile test is still more complex. However, within the limit of the experimental measurements and numerical analysis errors, the ENM based method would always achieve a more accurate description of the mechanical response of materials after necking formation and have tremendous application potential because of the following advantages: (a) the experimental $F-\Delta L$ curve is only required; thus tremendous amount of measurements during the whole process of tension can be avoided; (b) this method is program-controlled and easy to implement in a general commercial FE program and thus is more convenient in practice; (c) the iterative procedure is applicable to tensile test of both cylindrical and rectangular specimens; (d) the final effective true stress-strain curve can be determined for arbitrarily general strain hardening behavior of ductile metal as no specific analytical strain hardening model is assumed.

\section{Competing Interests}

The authors declare that they have no competing interests. 


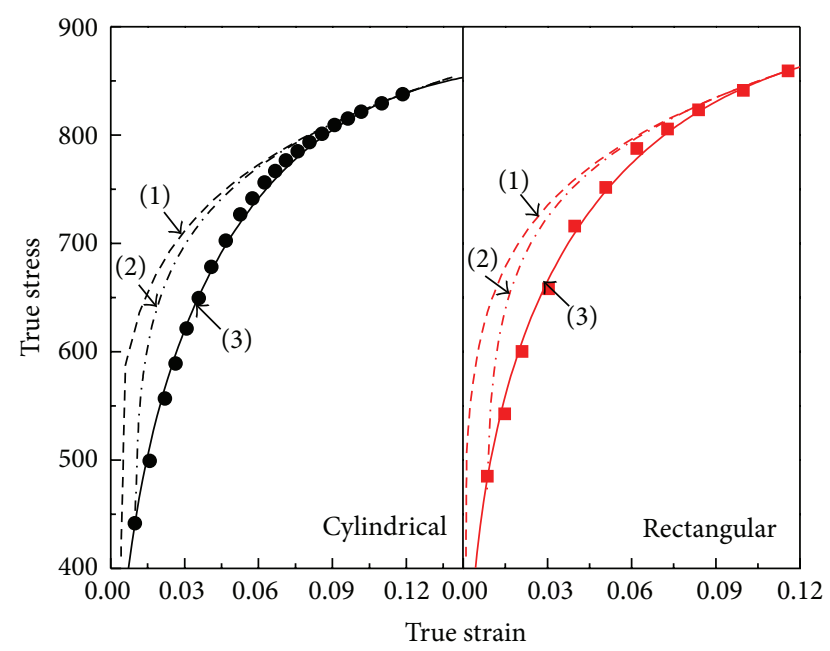

(a)

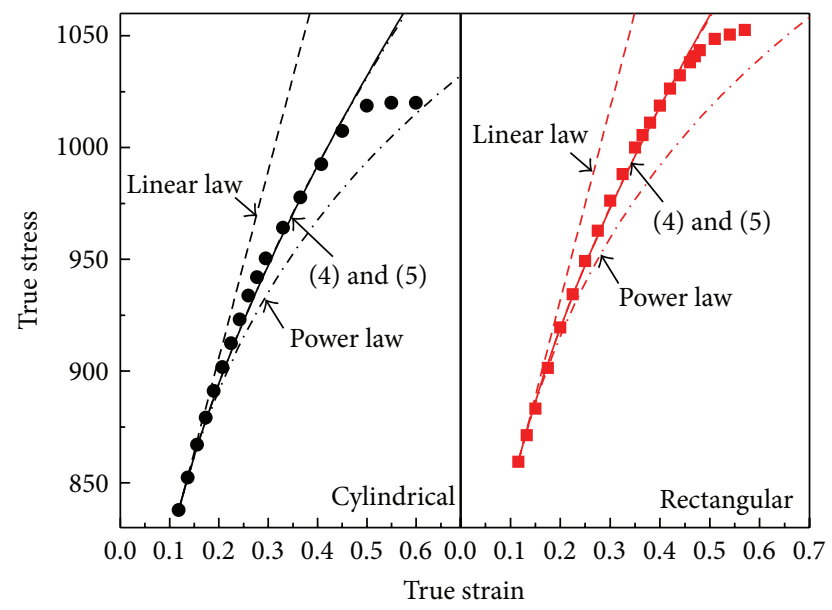

(b)

Figure 9: Fitting curves for different hardening laws: (a) before necking and (b) after necking.

\section{Acknowledgments}

This work is supported by the National Natural Science Foundation of China (Grant no. NSC-51378417), Program for Changjiang Scholars and Innovative Research Team in University (IRT13089), and PhD Innovation Fund of Xian University of Architecture and Technology.

\section{References}

[1] M. Joun, I. Choi, J. Eom, and M. Lee, "Finite element analysis of tensile testing with emphasis on necking," Computational Materials Science, vol. 41, no. 1, pp. 63-69, 2007.

[2] Z. L. Zhang, M. Hauge, J. Ødegård, and C. Thaulow, "Determining material true stress-strain curve from tensile specimens with rectangular cross-section," International Journal of Solids and Structures, vol. 36, no. 23, pp. 3497-3516, 1999.

[3] E. E. Cabezas and D. J. Celentano, "Experimental and numerical analysis of the tensile test using sheet specimens," Finite Elements in Analysis and Design, vol. 40, no. 5-6, pp. 555-575, 2004.
[4] S. Talbert and B. Avitzur, Elementary Mechanics of Plastic Flow in Metal Forming, John Wiley \& Sons, New York, NY, USA, 1996.

[5] J. C. Simo, "Numerical analysis and simulation of plasticity," in Handbook of Numerical Analysis, vol. 4, pp. 183-499, Elsevier Science, Amsterdam, Netherlands, 1998.

[6] X. Duan, M. Jain, D. R. Metzger et al., "A unified finite element approach for the study of postyielding deformation behavior of formable sheet materials," Journal of Pressure Vessel Technology, vol. 129, no. 4, pp. 689-697, 2006.

[7] W. Q. Shen and N. Jones, "Uniaxial true stress-true strain curve for a ductile metal," Nuclear Engineering and Design, vol. 140, no. 2, pp. 153-158, 1993.

[8] M. Brünig, "Numerical analysis and modeling of large deformation and necking behavior of tensile specimens," Finite Elements in Analysis and Design, vol. 28, no. 4, pp. 303-319, 1998.

[9] J. Kajberg and G. Lindkvist, "Characterisation of materials subjected to large strains by inverse modelling based on inplane displacement fields," International Journal of Solids \& Structures, vol. 41, no. 13, pp. 3439-3459, 2004.

[10] P. Bridgman, "The stress distribution at the neck of a tension specimen," Transactions of the American Society for Metals, vol. 32, pp. 553-574, 1944.

[11] P. Bridgman, Studies in Large Plastic and Fracture, McGrawHill, New York, NY, USA, 1952.

[12] Y. Ling, "Uniaxial true stress-strain after necking," AMP Journal of Technology, vol. 5, pp. 37-48, 1996.

[13] R. B. Joshi, A. E. Bayoumi, and H. M. Zbib, "The use of digital processing in studying stretch-forming sheet metal," Experimental Mechanics, vol. 32, no. 2, pp. 117-123, 1992.

[14] I. Scheider, W. Brocks, and A. Cornec, "Procedure for the determination of true stress-strain curves from tensile tests with rectangular cross-section specimens," Journal of Engineering Materials and Technology, vol. 126, no. 1, pp. 70-76, 2004.

[15] M. M. Ahmmad and Y. Sumi, "Strength and deformability of corroded steel plates under quasi-static tensile load," Journal of Marine Science and Technology, vol. 15, no. 1, pp. 1-15, 2010.

[16] A. C. Bannister, J. Ruiz Ocejo, and F. Gutierrez-Solana, "Implications of the yield stress/tensile stress ratio to the SINTAP failure assessment diagrams for homogeneous materials," Engineering Fracture Mechanics, vol. 67, no. 6, pp. 547-562, 2000.

[17] K. S. Zhano and Z. H. Li, "Numerical analysis of the stress-strain curve and fracture initiation for ductile material," Engineering Fracture Mechanics, vol. 49, no. 2, pp. 235-241, 1994.

[18] M. Joun, J. G. Eom, and M. C. Lee, "A new method for acquiring true stress-strain curves over a large range of strains using a tensile test and finite element method," Mechanics of Materials, vol. 40, no. 7, pp. 586-593, 2008.

[19] W. J. Dan, W. G. Zhang, S. H. Li, and Z. Q. Lin, "An experimental investigation of large-strain tensile behavior of a metal sheet," Materials and Design, vol. 28, no. 7, pp. 2190-2196, 2007.

[20] J. Gao, Research on materials test technology of real stress-strain based on machine vision [Ph.D. dissertation], Harbin Institute of Technology, Harbin, China, 2006 (Chinese).

[21] I. Barsoum and K. F. Al Ali, "A procedure to determine the tangential true stress-strain behavior of pipes," International Journal of Pressure Vessels and Piping, vol. 128, pp. 59-68, 2015. 
[22] J. Aronofsky, "Evaluation of stress distribution in the symmetrical neck of flat tensile bars," Journal of Applied Mechanics, pp. 75-84, 1951.

[23] C. García-Garino, F. Gabaldón, and J. M. Goicolea, "Finite element simulation of the simple tension test in metals," Finite Elements in Analysis and Design, vol. 42, no. 13, pp. 1187-1197, 2006. 

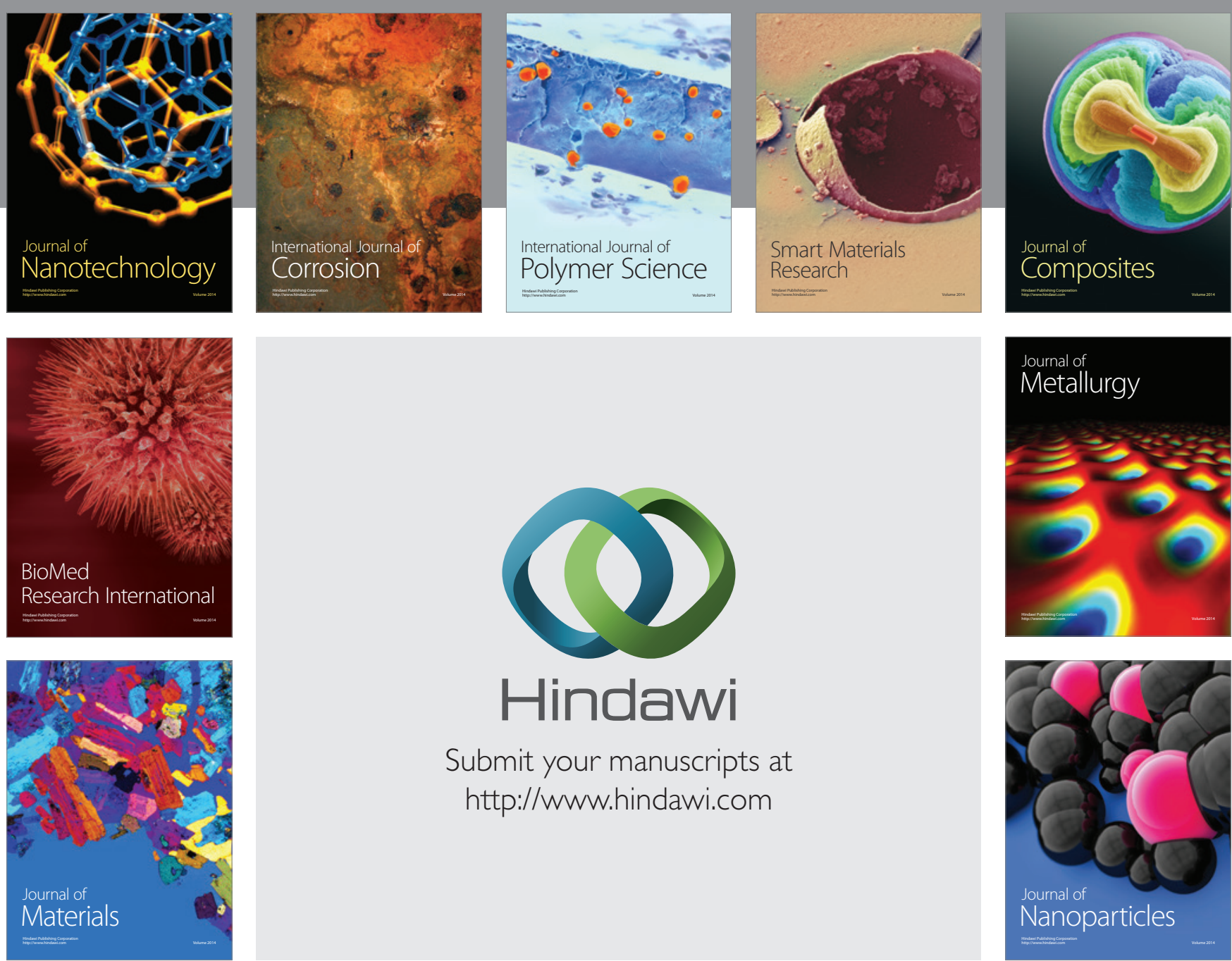

\section{Hindawi}

Submit your manuscripts at

http://www.hindawi.com

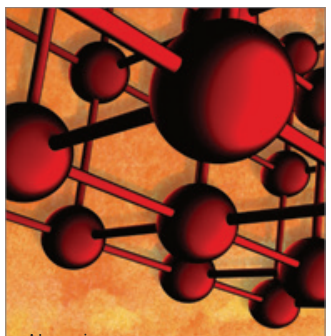

Materials Science and Engineering
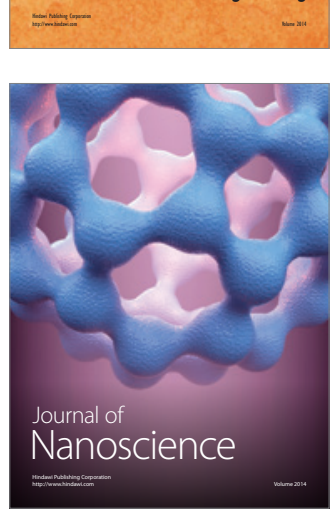
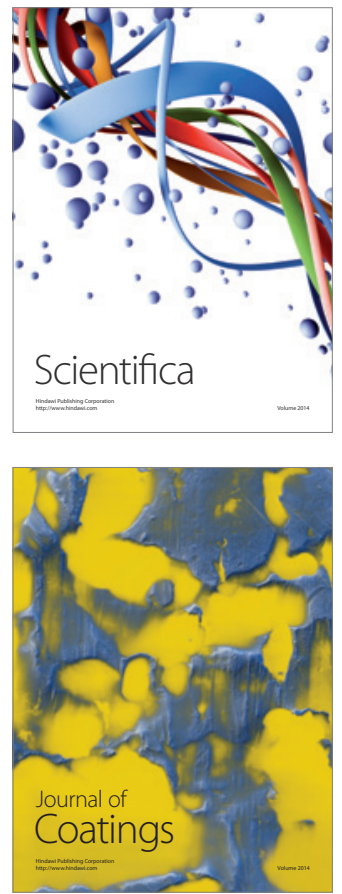
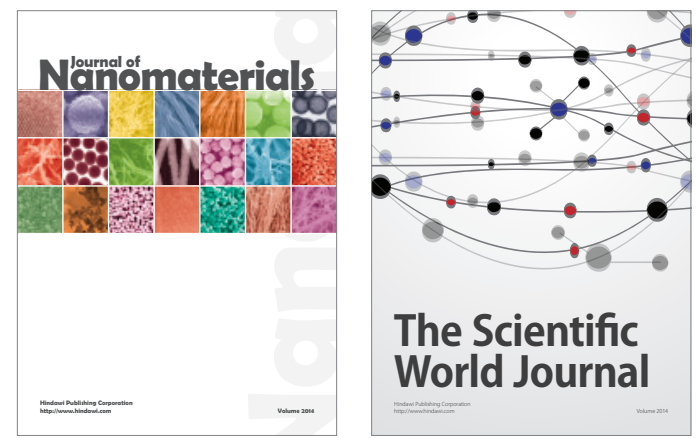

The Scientific World Journal
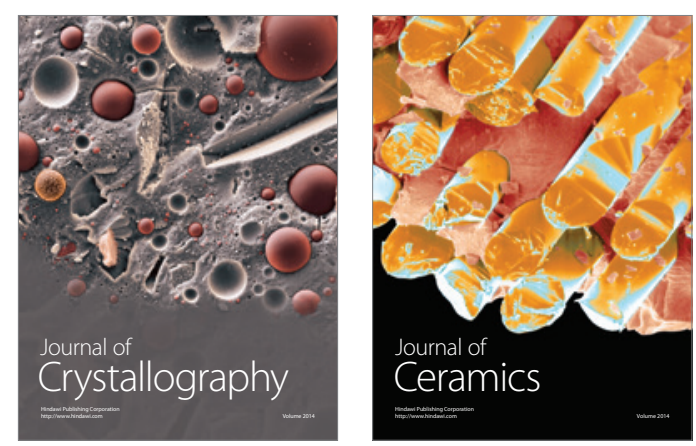
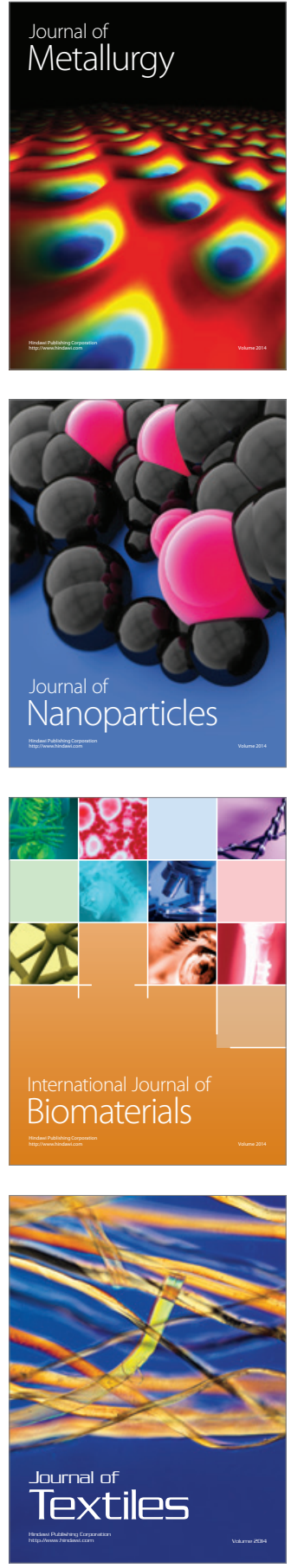